\title{
Sistema de gestión estratégica forestal participativa para el desarrollo forestal sustentable
}

\section{Strategic participatory forest management system for sustainable forestry development}

\author{
Concepción Luján-Álvarez*, Jesús Miguel Olivas-García', Susana Vázquez-Álvarez², \\ Javier Hernández-Salas' y Luis Ubaldo Castruita-Esparzal
}

Universidad Autónoma de Chihuahua. Facultad
de Ciencias Agrícolas y Forestales. Delicias,
Chihuahua., México. jolivas@uach.mx, jhernans@uach.mx.Icastruita@uach.mx

\author{
2 Consultor Forestal. Delicias, Chihuahua, México. * *Autor de correspondencia. clujanl2@hotmail.com \\ p76977@gmail.com
}

\section{RESUMEN}

Los impactos de la globalización económica se manifiestan en las dimensiones social, económica-financiera, institucional y ambiental, en una sociedad gobernada económicamente de arriba hacia abajo. En México, esos efectos han creado la necesidad de desarrollar con visión de sustentabilidad el sector forestal. El objetivo es establecer un sistema de gestión estratégica forestal participativa que impulse el desarrollo forestal sustentable. Este sistema de gestión es integral, sistemático, adaptativo, y se basa en el pensamiento estratégico, la visión holística y la democracia participativa, e incluye toma de decisiones de "abajo hacia arriba", con observancia de la normativa establecida. Se establece el sistema con el diseño, aplicación, acompañamiento multidimensional y seguimiento para el desarrollo forestal sustentable en el ejido El Largo y Anexos, Chihuahua, Mex. Se definieron cinco ejes de desarrollo: manejo forestal y biodiversidad, abastecimiento y producción forestal, desarrollo industrial, desarrollo de mercados y organización y administración. Como resultado, se han logrado avances en los diferentes ejes de desarrollo establecidos (e.g. mejoramiento de niveles organizacionales y administrativos), mejor nivel de productividad, buen manejo forestal, incremento de empleos y oportunidades de mercados, entre otros. En conclusión, el sistema se caracteriza por ser integral y dinámico diferente al modelo tradicional del desarrollo forestal en ejidos forestales. Promueve el desarrollo forestal participativo, anticipativo y autogestivo, ya que son los ejidos forestales quienes viven con los efectos de las acciones tomadas en su comunidad. Representa un cambio de paradigma integral; puede ser replicado a escalas local, regional y nacional en diferentes condiciones sociales, económicas y ambientales.

PALABRAS CLAVE: Chihuahua forestal, competitividad forestal, comunidad sustentable, modelo de gestión forestal, sistema socioecológico.

\section{ABSTRACT}

The impacts of economic globalization are manifested on the social, economic-financial, institutional and environmental, dimensions, in a society economically governedfrom the top-down. In Mexico, these effects have created the need to develop the forestry sector with sustainability vision. The objective is to present a participatory strategic forest management system that promotes sustainable forestry development. The strategic management system is integral, systematic, adaptive, and is philosophically based on strategic thinking, holistic vision, and participatory democracy. It includes "bottom-up" decision-making in compliance with the established regulations. The system is established with the design, application, multidimensional support, and monitoring for sustainable forestry development in the ejido el Largo y Anexos, Chihuahua, Mex. Five axes of development were defined: forest management and biodiversity, forest supply and production, industrial development, market development, and organization and management. As a result, progress has been made in the different axes of development established: improvement of organizational and management levels, better productivity, good forest management, increased employment, and market opportunities, among others. In conclusion, the system is characterized by being comprehensively different from the traditional model of forestry development in forest ejidos. It promotes participatory, anticipatory, and self-managed community forestry development, since the forest ejidos are those who live with the effects of the actions taken in their community. It represents a paradigm shift. It can be replicated at the local, regional, and national levels under different social, economic and environmental conditions.

KEYWORDS: Forestry Chihuahua, forest competitiveness, sustainable community, forest management model, socio-ecological system. 


\section{INTRODUCCIÓN}

La fuerte competencia en momentos en que la globalización tiene cada vez más auge e impacto, se manifiestan en la esfera financiera, energética, alimentaria, ambiental, institucional y hasta de valores de una sociedad gobernada económicamente de arriba hacia abajo por la economía de mercado (Giménez, 2008). Esta condición ha marcado tendencias para la concentración de esfuerzos en gestionar estratégicamente y de manera participativa el mejoramiento del desarrollo sustentable con visión de futuro. Sus fundamentos y filosofía no se han traducido en una ética con normas de conducta que reorienten los procesos de desarrollo tradicional económicos y políticos hacia una nueva racionalidad social y formas sustentables de producción y de vida, para poner fin a la pobreza y el hambre, y alcanzar el desarrollo rural sustentable (AriasHernández y Salazar-Sánchez, 2018).

En México, el Plan Nacional de Desarrollo 2019-2024 establece que la política social está comprometida a impulsar el desarrollo sostenible, un factor indispensable para el bienestar, orientado a satisfacer las necesidades de las generaciones presentes sin comprometer los recursos naturales que requerirán las generaciones futuras (Secretaría de Gobernación, 2019). En particular, la política forestal considera como eje fundamental el desarrollo forestal sustentable, toda vez que la gran proporción de la propiedad social de los bosques $(80 \%)$, pertenece a ejidos y comunidades forestales (EyCFs) (Instituto Nacional de Estadística, Geografía e Informática [Inegi], 2016). A la vez, como lo establece la Secretaría del Medio Ambiente y Recursos Naturales [Semarnat] y la Comisión Nacional Forestal [Conafor] (2019), esta política forestal es una respuesta al deterioro de los ecosistemas, mal aprovechamiento de los recursos forestales, disminución de su productividad y limitados beneficios sociales y ambientales, entre otros.

Para el ejercicio del desarrollo forestal sustentable, los territorios rurales se definen como espacios geográficos, con un tejido social específico, de una base de recursos naturales particular, con instituciones y formas de organización propias de acuerdo con su naturaleza de ejido o comunidad, así como de formas de producción, intercambio y distribución del ingreso. Esto, representa un sistema socioecológico (Sepúlveda, Rodríguez, Echeverría y Portilla, 2003; Palomo et al., 2010). En congruencia con lo mencionado, es necesario considerar el elemento multidimensionalidad, el cual da cuenta de los diversos componentes que conforman un sistema territorialsocioecológico comunitario, siendo estas dimensiones: a) económica, b) social-cultural, c) ambiental y d) político institucional.

Por otra parte, Chapela (2012) menciona que entre las principales debilidades del sector forestal se encuentran: a) la limitada inversión en la generación, transferencia y aplicación de conocimientos, b) la escasa visión empresarial, c) las capacidades administrativas y gerenciales deficientes, d) el rezago tecnológico y posibilidades limitadas para la adopción de tecnología en el sistema productivo forestal, entre otros.

En el México forestal, unos de los actores de base importantes para el desarrollo forestal sustentable, lo constituyen los ejidos y comunidades forestales (EyCF), mismos que hasta antes de 1992 representaron las dos modalidades del régimen de propiedad social. Sin embargo, después de que se hicieron las reformas de 1992 a la Ley Agraria, los ejidos y comunidades forestales fueron definidos con nuevas variantes o modificaciones a su régimen de propiedad que los hacen diferentes a su condición agraria anterior, toda vez que el ejido representa una modalidad de propiedad privada, mientras que la comunidad agraria se constituye en un modelo de propiedad en sí misma. A la vez, es importante mencionar que, para medios académico, legislativo, gubernamental, judicial, empresarial y campesino, entre otros; existe la idea de que su régimen jurídico conserva las características de la propiedad social (Pérez-Castañeda y Makinlay, 2015).

Se reconoce la importancia de EyCF, como un sistema socioecológico de gestión estratégica fundamental a escala nacional para transitar al desarrollo forestal sustentable (Inegi, 2016). Sin embargo, existen tres condiciones sociales que caracterizan la situación de los bosques comunitarios 
de México: la pobreza en que viven la gran mayoría de sus habitantes, el carácter social de su tenencia y la mayoría de las comunidades (75\%) aprovechan los bosques en condiciones de rentismo que puede en ocasiones ser sustentable y rentable (Conafor, 2008). Además, los EyCF enfrentan retos relacionados con su participación en los mercados de productos forestales debido a limitaciones en su nivel de competitividad y en procesos de certificación, entre otros, los cuales limitan su participación con mejores condiciones en el desarrollo forestal sustentable (Luján, Chapela, González, Olivas y Vázquez, 2018).

Los EyCF presentan niveles altos de marginación y pobreza en el país con grados de desarrollo precarios (Semarnat, 2014). Una alternativa para cambiar esto, es que las empresas forestales comunitarias deben transitar de un modelo de producción tradicional de cadenas productivas a la formación de cadenas de valor (Sánchez-Vidaña, Valtierra-Pacheco, González-Guillén y León-Merino, 2018). México debe avanzar sobre una base sólida en donde EyCF estén involucrados directamente en procesos de participación, autogestión estratégica y en toma de decisiones desde el diseño, aplicación, acompañamiento multidimensional, seguimiento y evaluación de planes, programas y proyectos de desarrollo forestal con enfoque holístico.

En atención a los cambios del año 1992 en la Ley Agraria, el régimen de propiedad del ejido y la comunidad presentan sus diferencias, el primero como una modalidad de propiedad privada y la segunda como un modelo de propiedad mixta o social-privada, incluyendo 4 instituciones jurídicas: la familia, las sucesiones, los contratos y el usufructo. Por lo tanto, esas diferencias de igual manera tienen un impacto en el comportamiento organizacional y de funcionamiento de la empresa forestal ejidal (EFE) y la empresa forestal comunitaria (EFC). Esto significa que, aun cuando en los regímenes de propiedad, procedimientos para la resolución de controversias y la estructura institucional de procuración de justicia agraria se registraron cambios radicales en la propiedad, ya han transcurrido más de 20 años y en algunos sectores se piensa que el régimen jurídico no varió gran cosa, por lo que se mantenían dentro de la propiedad social, toda vez que lo que se realizaba en la realidad era distinto a lo establecido en la ley. Esto da motivos para actitudes que no incentivan la eficiencia y la productividad, a menos que se apliquen políticas públicas y sistemas de gestión estratégica que contrarresten esos fenómenos (Pérez-Castañeda y Makinlay, 2015).

Por ello, en la EFE se evidencia un tipo de organización empresarial que, por su organización, toma de decisión y régimen de propiedad, tiende a manifestarse como modalidad de propiedad privada y la EFC está vinculada a una formación social particular, ya que: a) existe propiedad colectiva de los bosques (no propiedad privada); b) toma de decisiones consensuada en asamblea; y c) distribución colectiva de utilidades para una inversión social y productiva. Esto contribuye favorablemente a que los objetivos económicos no se desarticulen de los sociales y ambientales, favoreciendo a los miembros de la comunidad (Baños-Rosas y Lara-Rodríguez, 2013; Barrera, Cuervo, Hernández y Rodríguez, 2010). Los ejidos y comunidades forestales, así como las empresas forestales ejidales y comunitarias representan, per se, un sistema socioecológico complejo, por lo que es importante considerar los fundamentos teóricos y principios que sustentan el funcionamiento de un sistema de gestión estratégica como una alternativa para fortalecer el desarrollo forestal sustentable. Para ello, se conceptualiza a la gestión estratégica como el conjunto de acciones que se llevan a cabo para promover e impulsar el desarrollo de un sistema con una visión integral, e incluye la dirección y administración estratégica de la misma (Kandzior, 2013). Con base en ello, se presentan los fundamentos teóricos y principios estructurales que sustentan el diseño y puesta en marcha de un sistema de gestión estratégica participativa para el desarrollo sustentable considerando los sistemas y bases siguientes (Kandzior, 2013; Ramírez, 1999; Palomo et al., 2010): 


\section{i) Pensamiento estratégico}

La cultura organizacional y la comunitaria representan el pensamiento colectivo a través de compartir sus ideas, puntos de vista, tradiciones, costumbres, creencias, valores, actitudes, percepciones y vivencias del mundo que los rodea. La comunidad deberá aprender a integrarse y monitorear continuamente el ambiente para formular, o en su caso, reformular estrategias que sustenten la visión o rumbo que desea tomar la comunidad forestal para orientar su desarrollo forestal sustentable, y así cambiar de comunidad reactiva y/o adaptativa a comunidad proactiva y/o anticipativa.

\section{ii) Visión holística}

El concepto holístico permite ver la realidad como un todo. Esto facilita ver a las organizaciones, ejidos o comunidades forestales como un todo que ayuda a responder las siguientes interrogantes: ¿Cuál es su razón de ser? ¿Cuál es su propósito y fin? ¿Cuáles son sus funciones y su interrelación en sus componentes?, entre otras. El holismo considera que el todo es mayor que la suma de sus partes, debido a que la organización o comunidad total proporciona las propiedades adicionales que conceptualizan el todo (Ramírez, 1999).

\section{iii) Democracia participativa}

La participación de los actores de base del ejido o comunidad forestal deben de estar involucrados en la definición de su futuro deseable y alcanzable, a través de un proceso democrático participativo, que les permita la toma de decisiones de "abajo hacia arriba", de acuerdo con sus intereses, necesidades y aspiraciones, tomando en consideración la normativa existente. Lo anterior es muy importante, debido a que es la gente de la comunidad u organización quien vive con los efectos y consecuencias de cualquier acción llevada a cabo en su comunidad. Por tal razón, es la gente de la propia comunidad, ejido u organización quien mejor conoce sus condiciones de vida, a través del tiempo, así como la interrelación que ha tenido con los recursos naturales y el ambiente, por lo que ellos necesariamente deben de tener la oportunidad de su involucramiento efectivo en la toma de decisiones a favor del desarrollo de su comunidad.

Lo anterior conduce a la necesidad de la aplicación de un sistema de gestión estratégica forestal participativa para el desarrollo forestal sustentable que favorezca su visiónacción, como la propuesta que ahora se presenta, para el fortalecimiento de capacidades en estructura organizacional, poder de gestión y gobernanza con un tejido específico fortalecido con formas de organización propias (Palomo, Martín-López, López y Montes, 2010; Carroz, 2005), ya que son ellos quienes viven con las consecuencias inmediatas de cualquier acción o decisión sobre sus recursos forestales y el ejido forestal.

Algunos casos realizados en ejidos forestales sirvieron de base y contribuyeron para la integración del sistema de gestión destacando los siguientes: i) Plan estratégico participativo para el desarrollo forestal sustentable del estado de Chihuahua (Luján, Olivas, González, Gómez y Cuautle, 2006) y ii) Desarrollo forestal sustentable en el estado de Nuevo León (Capó, Luján, Treviño, Nájera y Morales, 2007).

\section{OBJETIVOS}

Establecer un sistema de gestión estratégica forestal participativa en el ejido El Largo y Anexos, Chihuahua, Mex. para impulsar el desarrollo forestal sustentable con visión holística en México.

\section{MATERIALES Y MÉTODOS}

A continuación, se presentan las características del proceso sistemático seguido para el diseño, aplicación, seguimiento y evaluación del sistema de gestión estratégica participativa para el desarrollo forestal sustentable:

\section{Establecimiento de las etapas estructurales del proceso de gestión estratégica participativa}

La dinámica de cambios continuos y sus impactos en la interacción del sistema socio económico y ecológico, compromete a los EyCF a gestionar estratégicamente, de forma sistemática y participativa fortaleciendo sus capacidades para impulsar el desarrollo forestal sustentable 
con visión de futuro. Por ello, las etapas del proceso de gestión estratégica participativa están estructuradas sistemáticamente en un proceso continuo, sistemático, adaptativo y con visión de futuro, siendo las que se ilustran en la figura 1 (Luján, Olivas y Vázquez, 2019):

Las etapas del proceso de gestión estratégica participativa son las siguientes:

Etapa 1. Socialización-inducción: Se refiere a la necesidad de la socialización, con la comunidad, de la naturaleza y alcances de un proyecto de desarrollo comunitario, incluye: inducción, sensibilización y compromiso comunitario e institucional.

Etapa 2. Diseño del proyecto: ejecución de la planeación estratégica participativa para la obtención del plan rector y plan operativo para la puesta en marcha del proyecto de desarrollo comunitario. Un componente fundamental de la estructura de esta etapa es la aplicación de la metodología de planeación participativa denominada "Conferencia de Búsqueda" y "Multiconferencia de Búsqueda" (Emery y Ronald, 1996), como una estrategia integral dinámica, flexible y adaptable a diferentes ambientes y territorialidades. Se basa en la teoría de sistemas abiertos y en procesos de toma de decisiones de "abajo hacia arriba", diseño de escenarios más probables y deseables y facilita la obtención de planes rectores estratégicos, acompañamiento y seguimiento para el desarrollo comunitario (Goodstein, Nolan y Pfeiffer, 2002; Luján, Olivas y Vázquez, 2011).

Etapa 3. Fortalecimiento del capital social y humano: necesidad de mejorar las capacidades del capital social, humano, tejido social y capacitación para la organización, que le permita la implementación, dirección, control y seguimiento del proyecto.

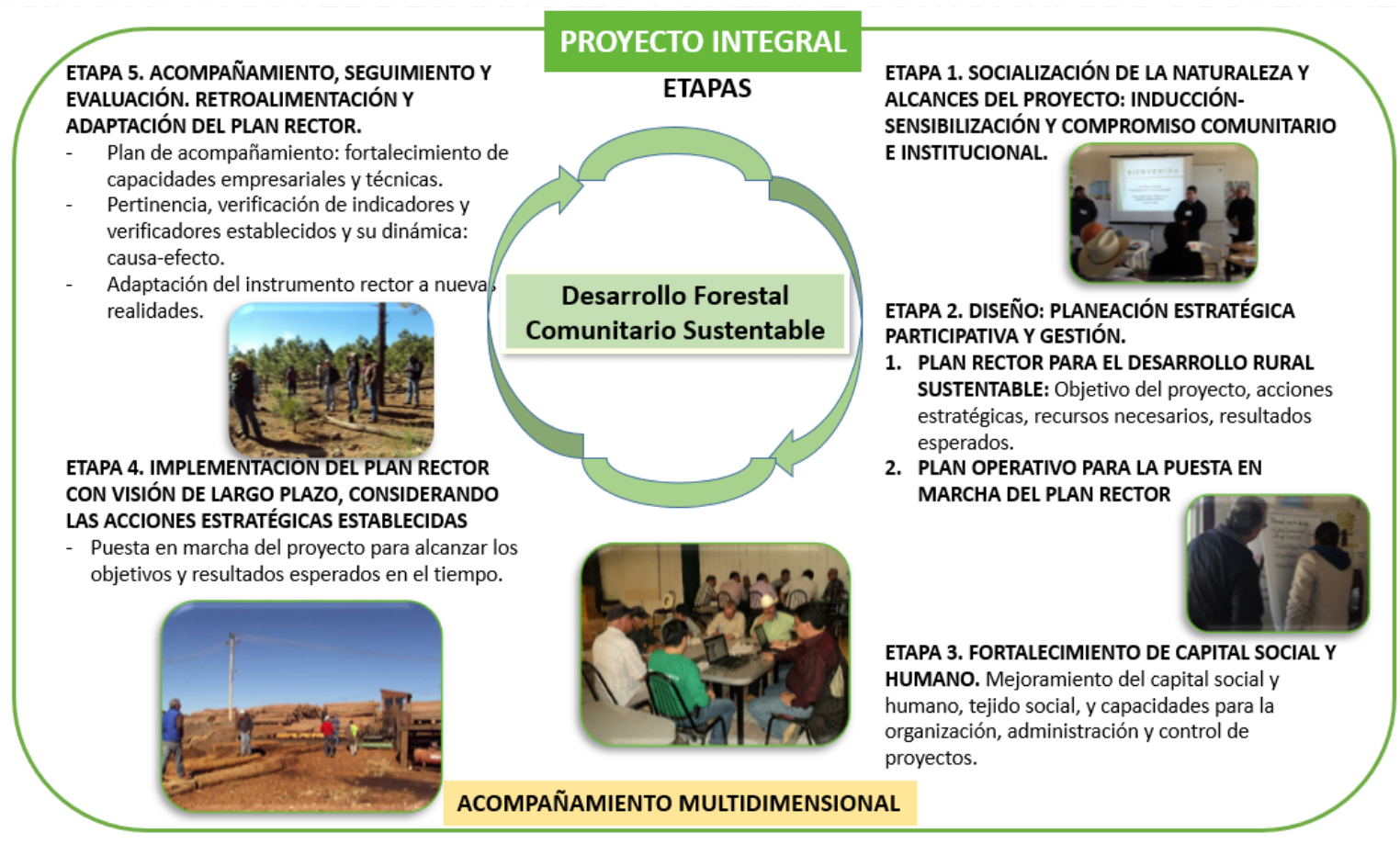

FIgura 1. Componentes/Etapas estructurales del proceso de gestión estratégica participativa para el desarrollo forestal sustentable (Luján et al., 2019). 
Etapa 4. Puesta en marcha del plan rector y plan operativo con visión de largo plazo para lograr los objetivos y resultados esperados del desarrollo de la comunidad.

Etapa 5. Acompañamiento multidimensional, seguimiento y evaluación, adaptación. Realización de procesos de retroalimentación y adaptación/ajustes del plan rector y plan operativo, en su caso, para lograr un proceso continuo, dinámico, adaptativo y con visión holística y así sucesivamente se continúa el ciclo del proceso.

\section{Diseño, aplicación, seguimiento y evaluación del sistema de gestión estratégica forestal participativa}

Se procedió al diseño, aplicación, validación, aprobación por la Asamblea General, así como la transferencia, adopción, así como la posterior aplicación y evaluación del sistema de gestión estratégica participativa para el desarrollo forestal sustentable y empresa forestal ejidal. Se tomó como base y caso de estudio la ejecución del proyecto de desarrollo forestal integral del Ejido el Largo y Anexos, municipio de Madera, Chih., que es el ejido forestal más grande en superficie y producción forestal en México (Hodgdon y Estrada, 2015). El proceso integral de gestión estratégica forestal participativa se llevó a cabo durante el período del año 2011 al 2015. El procedimiento consistió en las acciones siguientes:

\section{a) Definición del ejido forestal: Ejido El Largo y Anexos}

El ejido se localiza en el municipio de Madera, estado de Chihuahua. Sus coordenadas geográficas son $29^{\circ} 41^{\prime} 16^{\prime \prime N ~ y ~}$ 108¹6'19"W, con una altitud media de 2140 msnm (Fig. 2).
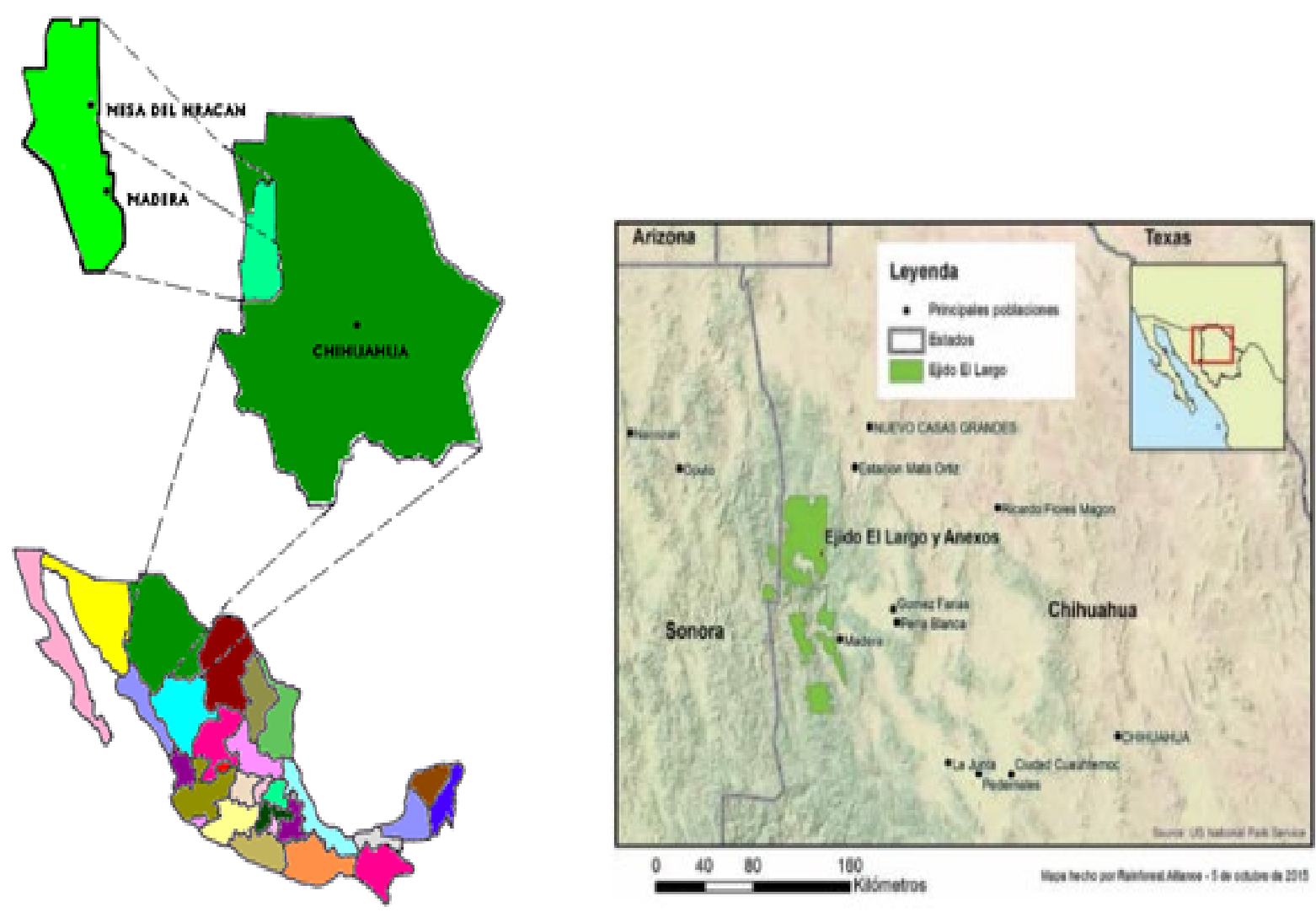

Figura 2. Ubicación del Ejido el Largo y Anexos, Mpio. Madera, Chih. (Luján et al. 2011; Hodgdon y Estrada, 2015). 
El ejido comprende una superficie de 261460 ha y está integrado por un total de 1723 ejidatarios con sus derechos legalmente reconocidos. El ejido tiene autorizada una corta anual de más de 315000 metros cúbicos $\left(290804 \mathrm{~m}^{3}\right.$ rta de pino y $25738 \mathrm{~m}^{3} \mathrm{rta}$ de encino), lo que representa $28 \%$ del total de la producción del estado de Chihuahua y $4.6 \%$ de la producción nacional de México. Cuenta con la certificación forestal FSC desde marzo 2018. En un año promedio emplea a más de 2600 personas (Hodgdon y Estrada, 2015). Los mismos autores indican que aún con esas cualidades, el ejido enfrenta desafíos entre los que destacan: la discontinuidad de los liderazgos, débil capacidad administrativa y organizacional, maquinaria obsoleta e infraestructura que genera ineficiencias, falta de acceso al crédito y escasa penetración de los mercados.

El ejido se propuso realizar importantes cambios en su dinámica del desarrollo integral, entre ellos los siguientes: a) modernización y diversificación de la industria, b) aprovechamiento de subproductos de asierre que actualmente son desperdicios, para bajar costos e incrementar ingresos, c) elaboración y comercialización de productos de arbolado delgado, d) mejoramiento de la organización y administración estratégica, e) optimización del abastecimiento forestal y f) aprovechamiento del potencial de los recursos, a la par, que se mantenga y/o mejore el manejo forestal y la biodiversidad.

\section{b) Socialización e inducción de la planeación estratégica participativa}

Se realizó un proceso de socialización, sensibilización e inducción y una visita técnica exploratoria que incluye áreas industriales y de campo para retroalimentar la necesidad de impulsar el desarrollo integral del ejido y la importancia de contar con acompañamiento interinstitucional en el proceso. Se identificaron los siguientes ejes de desarrollo del ejido con visión integral: a) Manejo forestal y biodiversidad, b) Abastecimiento y producción forestal, c) Desarrollo industrial, d) Desarrollo de mercados y e) Administración y organización empresarial.

Las actividades se realizaron con la participación de las autoridades ejidales y un grupo de expertos externos representantes de las instituciones siguientes: Conafor; Proyecto Global Environmental Fund [GEF]; Rainforest Alliance; Gobierno del Estado de Chihuahua - Dirección General Forestal; Gerencia Estatal de Chihuahua de la Conafor; FIRA; Reforestamos México, A.C. y la Facultad de Ciencias Agrícolas y Forestales de la Universidad Autónoma de Chihuahua [FCAyF-UACH]. Esta última fungió como equipo institucional coordinador y facilitador técnico del proyecto.

\section{c. Elaboración del plan operativo de trabajo para la definición de ejes de desarrollo con visión holística}

La elaboración del plan operativo se realizó de forma participativa (total 60 participantes), por medio de talleres y plenarias (3), y en cada uno de los ejes de desarrollo mencionados (12 participantes por eje). Su objetivo fue diseñar un plan de trabajo a seis meses. Este proceso se llevó a cabo con la participación de la Dirección Técnica Forestal del ejido, representantes expertos de las diferentes instancias involucradas y con el equipo coordinador y facilitador del proyecto (UACH-FCAyF) (Luján, Olivas y Vázquez, 2011).

d) Elaboración del diagnóstico actual y propuesta de mejora de los ejes de desarrollo y estrategias de seguimiento

Se elaboraron tanto el diagnóstico actual y la propuesta de mejora de cada uno de los ejes de desarrollo. Se identificaron fortalezas, debilidades y áreas de oportunidad, así como la integración de una propuesta de mejora por cada eje de desarrollo.

\section{e) Elaboración del plan rector y el plan operativo anual}

Se elaboraron el plan rector y plan operativo anual, en talleres participativos, estableciendo la misión y visión del ejido, así como sus objetivos estratégicos, acciones estratégicas, responsables, temporalidad y verificadores/ evidencias de cumplimiento. Se contó con la participación de autoridades ejidales, dirección técnica forestal del ejido, expertos externos de instancias colaboradoras responsables de los ejes de desarrollo, grupos ejidales, e integrantes del 
equipo coordinador. Posteriormente, se presentó y validó el contenido del plan rector con visión de largo plazo y el plan operativo anual para la puesta en marcha del proyecto para el desarrollo forestal integral del ejido. Finalmente, se promovieron procesos de sensibilización y motivación para la apropiación y compromiso del ejido en la implementación del proyecto, donde se definieron acuerdos y compromisos de seguimiento en la Asamblea General (Luján et al., 2011).

\section{f) Presentación, validación final, transferencia $y$ adopción}

Las autoridades ejidales convocaron a los ejidatarios legalmente reconocidos, a una Asamblea General con el propósito de presentar y aprobar el plan rector con visión a largo plazo y el plan operativo anual, mismo que fue aprobado por los asambleístas presentes por unanimidad. Adicionalmente, las instituciones participantes hicieron patente su decisión de seguir brindando el apoyo al ejido en la implementación del proyecto.

\section{g) Implementación, seguimiento y evaluación}

Se integró un grupo interinstitucional con el objeto de aplicar el sistema de gestión estratégica forestal participativa para el desarrollo comunitario sustentable y de empresas comunitarias. Se inició con el diseño y la aplicación de un sistema de organización y administración con visión empresarial a largo plazo, siendo prioritaria su realización para favorecer la implementación del plan rector. Se da seguimiento a su aplicación, acompañamiento multidimensional, evaluación y control. Estas acciones se realizaron de acuerdo con lo programado y aprobado por la Asamblea General de ejidatarios basados en el sistema de gestión estratégica forestal establecido durante el período del año del 2011 al 2015 (Hodgdon y Estrada, 2015).

\section{RESULTADOS Y DISCUSIÓN}

Como resultado de la aplicación del sistema de gestión forestal, se estableció un sistema de gestión integral que incluye la interacción entre el sistema Ejido Forestal, empresa forestal ejidal y su contexto multidimensional para la competitividad, sustentabilidad, así como su dimensión dinámica en sus componentes, contextos y en el tiempo. Presenta cualidades en su estructura y operación que lo hacen ser original con identidad ejidal (Fig. 3). Adicionalmente, se sustenta en la teoría de sistemas abiertos (Ramírez, 1999), toda vez que está integrado por componentes que interactúan entre sí por medio de los ejes de desarrollo definidos. A la vez, promueve apropiación y compromiso con actitud proactiva de los actores locales que integran el ejido forestal para impulsar el desarrollo forestal sustentable, de acuerdo con la integración y funcionamiento de los ejes de desarrollo del ejido que se tomaron como base estructural y con visión multidimensional y multipropósito.

Por lo tanto, como lo establecen Hodgdon y Estrada (2015), el diseño, aplicación, validación, aprobación, transferencia, adopción, implementación y evaluación están sustentados en que este sistema de gestión estratégica se diseñó y se puso en marcha para la conducción del proyecto para el desarrollo forestal integral como un caso de estudio y generando beneficios socioeconómicos y ambientales del año 2011 al año 2015.

La puesta en marcha, el seguimiento y la evaluación del sistema de gestión forestal mencionado permitieron lograr avances en los diferentes ejes de desarrollo (5) y su transversalidad, como lo indican Hodgdon y Estrada (2015), lo cual ratifica en la temporalidad y espacio, las cualidades del diseño y aplicación del sistema de gestión estratégica generado e implementado a favor del desarrollo forestal integral y de la empresa forestal comunitaria con visión de sustentabilidad (Fig. 3). De tal manera que es necesario tomar en consideración que los resultados que se han obtenido en el período de implementación y evaluación indicados no pueden ser establecidos como productos finales, sino más bien como el inicio de un proceso sistémico y dinámico, que incluye análisis y discusión teóricos, metodológicos y éticos respecto al desarrollo futuro del ejido forestal como un sistema abierto (ClarkTapia, Fuente-Carrasco, Corrado, Ramos-Morales y Aguirre-Hidalgo, 2018). 


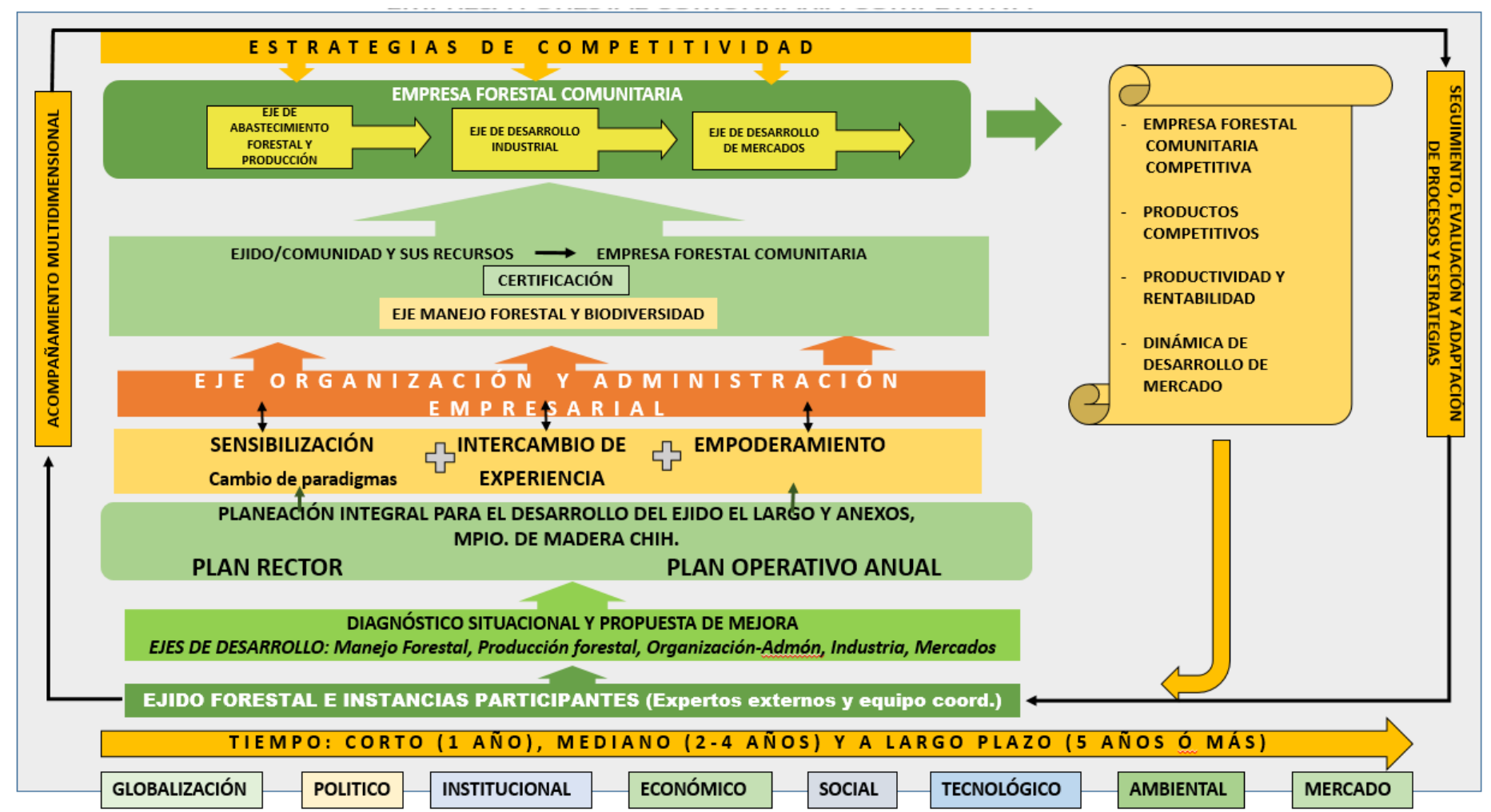

FIGURA 3. Sistema de gestión estratégica forestal participativa para el desarrollo forestal sustentable y empresa forestal ejidal (Lujan et al., 2011).

Como evidencia de los avances en la implementación, los ejes de desarrollo que han mostrado progreso son principalmente el de organización y administración, manejo forestal y biodiversidad, desarrollo industrial y desarrollo de mercados.

En el manejo forestal se realizaron estudios técnicos para la elaboración de un nuevo programa de manejo, existe incremento en la productividad forestal y se encontró que $85 \%$ de los ingresos registrados en el ejido vienen del manejo forestal. En el desarrollo organizacional y administrativo, se rediseñó la estructura organizacional y administrativa del ejido, así como la creación de un nuevo consejo administrativo para asegurar la continuidad en la gestión del ejido.

La estrategia es que con el tiempo se convierta en un consejo directivo permanente para atender el proyecto de desarrollo integral del ejido y fortalecer los procesos de gobernanza y administración holística. Así, se busca que los cambios periódicos de autoridades ejidales no limiten la ejecución continua de los procesos de desarrollo y favorecer que las decisiones y los procesos se pueden llevar a cabo a través de los cambios de liderazgo. En el desarrollo de mercados, se logró diversificar la cartera de clientes y penetrar mercados con valor agregado.

Referente al desarrollo industrial, se instaló una nueva planta de aserrío y de tableros. También se avanzó en la construcción de la planta industrial propuesta. Para esto, se realizó la gestión financiera por el ejido con la Financiera Nacional de Desarrollo Agropecuario, Rural, Forestal y Pesquero (FND) para la obtención del crédito para tal fin. Esta situación contrasta con lo establecido por Garibay (2007), quién indica que no todos los ejidos que tienen recursos forestales han podido desarrollar una empresa forestal exitosa. Esto, a pesar de que muchos ejidos han 
recibido apoyos gubernamentales para adquirir maquinaria para industrializar o para comercializar sus productos forestales. Esta gestión financiera fue una de las tareas clave para impulsar el desarrollo forestal integral del ejido.

En suma, se han logrado avances en los diferentes ejes de desarrollo establecidos con visión multidimensional: el mejoramiento de niveles organizacionales, administrativos y niveles de productividad y buen manejo forestal, gracias al programa de manejo forestal y biodiversidad con sus buenas prácticas marcadas por los estándares del Forest Stewardship Council (FSC). En relación con empleos se logró un incremento de 12\% entre el año 2011 y 2013, significando un incremento de aproximadamente 300 empleos directos, de los cuales algunos empleos fueron ocupados por mujeres pasando de 42 a 69, siendo un incremento de $62 \%$, favoreciendo el bienestar socioeconómico. Por lo tanto, la experiencia de la Empresa Forestal Ejidal más grande del mundo (Ejido el Largo y Anexos), con un volumen de cosecha equivalente al de una nación tropical, con 10 aserraderos y miles de empleados, resulta ser relevante para la mayoría de las empresas ejidales, ya que el mejoramiento de la eficiencia de la empresa forestal ejidal beneficia a 1839 familias (Hodgdon y Estrada, 2015).

Del sistema de gestión estratégica forestal participativa puesto en marcha resaltan las siguientes cualidades:

a) Se integró a los actores de base locales/ejidatarios, la participación de instituciones gubernamentales y no gubernamentales como expertos externos en los ejes de desarrollo ya mencionados, así como un equipo facilitador/coordinador técnico, en los procesos de toma de decisiones de "abajo hacia arriba". Esto representa en su estructura y operación un acompañamiento multidimensional e interdisciplinario, lo cual es una fortaleza del sistema de gestión forestal, aun cuando existe el riesgo, como es el caso del modelo tradicional y lo indicado por Sabogal et al. (2009), de que las organizaciones expertas/facilitadores y acompañantes de apoyo abandonen su papel de facilitación y se retiren en un tiempo determinado. El equipo de acompañamiento asumió que los expertos externos desarrollen una actitud de respeto y valorización de las capacidades de los actores locales para definir sus propios caminos de desarrollo según sus intereses, siendo así, finalmente una fortaleza del sistema.

El grupo de expertos externos institucionales y el equipo técnico facilitador, con el objeto de asegurar continuación en los procesos de desarrollo, tuvieron a bien capacitar a ejidatarios claves sugeridos por ellos mismos en los diferentes ejes de desarrollo para dar seguimiento en el futuro a las acciones estratégicas establecidas en el plan rector. Esto se realizó con el propósito de que, si los expertos externos tuvieran que abandonar al proceso, no existiera una dependencia absoluta de ellos en la organización y funcionamiento del ejido.

b) Tradicionalmente, en los procesos de toma de decisiones existe predominancia de los actores externos con liderazgos, sin respetar las necesidades, deseos e intereses de la gente local, así como la falta de mecanismos efectivos de acompañamiento multidimensional, la accesibilidad solamente para pocos productores forestales que participan en los proyectos y la falta de continuidad de las iniciativas (Sabogal et al., 2009). El sistema de gestión estratégica forestal incluye en su estructura y funcionamiento acciones estratégicas que promueven la participación de base aplicando el principio de la democracia participativa, respetando las demandas de los ejidatarios, teniendo en observancia la normatividad correspondiente y promoviendo así un desarrollo de base con apropiación y compromiso y de forma sistémica.

c) El sistema de gestión estratégica integra bases filosóficas (pensamiento estratégico, visión holística y democracia participativa), componentes, estructura/funcionalidad, diseño e implementación que sustentan los siguientes hechos: i) promueve el acompañamiento multidimensional por expertos externos y facilitadores al ejido, para que los ejidatarios diseñen y pongan en marcha sus propios proyectos de desarrollo, tomando en consideración la política forestal y normativa existente, entre otros factores, sin que los expertos externos tomen el liderazgo de los procesos no privilegiando las necesidades, intereses y deseos de los actores locales y su contexto y ii) promoción de mecanismos locales de aprendizaje para desarrollar la capacidad adaptativa de la gente local, según su 
conocimiento práctico, habilidades y destrezas (Giddens, 1984).

d) Desde el punto de vista de la empresa forestal, en general, ha tenido dependencia de las políticas y programas de apoyo gubernamental, además de situaciones internas de organización y capacidades del recurso humano, entre otras (Bray, 2007). Sin embargo, el sistema de gestión estratégica forestal aplicado promueve el desarrollo integral del ejido, con una vinculación estructural, operativa, técnica y administrativa que enlaza los ejes de desarrollo interno con visión holística. De esta manera, no da lugar a que un limitado consenso comunitario conduzca a una administración inadecuada (Rodríguez et al., 2019).

e) El sistema de gestión estrategia impulsa el empoderamiento del ejido o comunidad y permite fortalecer las capacidades del recurso humano (Janja et al., 2016), gracias al acompañamiento multidimensional facilitado por los expertos externos y el equipo técnico coordinador. Asimismo, el sistema de gestión representa una ventaja por la relación entre el ejido y la empresa ejidal (Janja et al., 2016). Esta ventaja es que el mismo ejido ejerce cierto control sobre la EFE y evita que algunos ejidatarios se adueñen de los recursos de la comunidad, además, de que el ejido puede decidir sobre los recursos económicos generados por el manejo forestal comunitario.

f) El sistema de gestión estratégica forestal, en coincidencia con lo que establece la Cooperación Alemana [GIZ] (2013), es renovador en el contexto del desarrollo forestal sustentable y de empresa forestal ejidal, toda vez que fomenta el manejo forestal sustentable e integración vertical del ejido. Hodgdon y Estrada (2015), quienes realizaron su investigación de seguimiento respecto a la aplicación sistema de gestión estratégica para el desarrollo integral del ejido el Largo y Anexos, mencionan que el ejido ha logrado una estructura organizacional y gobernanza que le ha permitido desarrollar acciones estratégicas para su integración. Los mismos autores hacen patente el progreso que se ha tenido como producto de la aplicación del sistema de gestión forestal, toda vez que se ha impactado en diferentes ejes de desarrollo del ejido como son: i) manejo forestal sustentable: incremento en producción $\mathrm{y}$ productividad forestal, mejora en conservación y protección de la biodiversidad y recursos asociados, ii) abastecimiento forestal: mayor eficiencia abastecimiento, incluyendo el derribo, el arrastre y el transporte, iii) desarrollo industrial: modemización de la industria forestal, iv) mercados y comercialización: diversificar la cartera de clientes y penetrar mercados con valor agregado y v) organización y administración: reingeniería organizacional, mejoramiento en el nivel de empleos, seguridad social, mejora en procesos administrativos, generando beneficios económicos, ecológicos y sociales que contribuyen a mejorar la calidad de vida de los pobladores del ejido.

g) El sistema de gestión estratégica impulsa el desarrollo ejidal con una visión sistémica, lo cual facilita un proceso continuo, sistemático y adaptativo. Esto coincide con Torres y Graft (2014), que indican que la gestión adaptativa no puede seguir siendo una administración de un bien o conjunto de bienes o servicios del bosque, bajo una estructura tradicional y manteniendo condiciones que no brindan los beneficios a los que se aspira. Por el contrario, debe ser una gestión realmente adaptativa en diversificación, niveles de integración y formas organizacionales con capacidad de respuesta a los cambios multidimensionales en el entorno.

\section{CONCLUSIONES}

El sistema de gestión forestal es integrador y representa ventajas sociales, económicas y ambientales para promover el desarrollo forestal sustentable y desarrollo de la empresa forestal ejidal.

La utilidad y pertinencia del sistema de gestión radica en su capacidad de apoyo a las decisiones locales, basadas en acciones e innovaciones asociadas al fomento de un desarrollo forestal sustentable.

La estructura y aplicación del sistema de gestión representa una estrategia integral que impulsa la promoción de cambios de paradigmas en el gobierno local para el desarrollo forestal sustentable con base participativa, autogestiva, emprendedora, competitiva y sustentable.

El sistema de gestión estratégica forestal participativo puede ser replicado y adaptado a escalas local, regional y 
nacional en diferentes condiciones socio-ecológicas, culturales, económicas y ambientales.

El sistema de gestión estratégica forestal es incluyente, ya que se sustenta en la alianza de carácter ejidal-públicoprivada, a través de la cual se articulan los actores locales y externos con presencia e incidencia para impulsar el desarrollo forestal integral con criterios de sustentabilidad y competitividad.

\section{REFERENCIAS}

Arias-Hernández, A., \& Salazar-Sánchez, J. L. (2018). Visión social del desarrollo rural sustentable. Colección: Estudios e Investigaciones Centro de Estudios para el Desarrollo Rural Sustentable y la Soberanía Alimentaria. Cámara de Diputados, LXIII Legislatura. Recuperado de http://www.cedrssa.gob.mx/files/b/8/60Visi $\% C 3 \%$ B3n_social _desarrollo_rural_sustentable.pdf

Barrera, J. M., Cuervo, S., Hernández-Corral, J. T., \& Rodríguez, J. L. (2010). Manual de buenas prácticas en aserraderos de comunidades forestales. Consejo Civil Mexicano de Silvicultura Sostenible, A.C., Rainforest Alliance y Reforestamos México. Recuperado de http:/ /www.ccmss.org.mx/wp-

content/uploads/2014/10/Manual_de_buenas_practicas_en_as erraderos_de_comunidades_forestales.pdf

Baños-Rosas, M., \& Lara-Rodríguez, R. (2013). Empresas forestales comunitarias y cooperativismo autogestionario: una aproximación teórico-práctica. Iberoamérica, 15(1) 237- 270.

Bray, D. (2007). El manejo comunitario de los bosques en México: veinte lecciones aprendidas y cuatro senderos para el futuro. En D. Bray, L. Merino, \& D. Barry eds. Los bosques comunitarios en México (pp. 417-434). Cd. de México. INE-Semarnat.. Recuperado de https://micrositios.inecc.gob.mx/publicaciones/libros/532/cap 14.pdf

Capó, M., Luján, C., Treviño, J. E., Nájera, J. A., \& Morales, L. (2007). Plan Estratégico para el esarrollo Forestal Sustentable en el estado de Nuevo León. Universidad Autónoma Agraria Antonio Narro-Saltillo, Coah. Monterrey, NL. Mex. Recuperado de http://www.conafor.gob.mx:8080/documentos/docs/12/186Pr ograma $\% 20$ Estrat $\%$ c3\%a9gico $\% 20$ Forestal $\% 20$ del $\% 20$ Estado $\%$ $20 \mathrm{de} \% 20$ Nuevo $\% 20 \mathrm{Le} \%$ c3\%b3n.pdf

Carroz, U. D. (2005). Modelo de gestión estratégica para el desarrollo de capacidades tecnologicas. DAC-UCLA, USA. Recuperado de http://www.ucla.edu.ve/dac/compendium/Revista15/DCarroz .pdf
Chapela, G. (2012). Problemas y oportunidades en el mercado para las empresas sociales forestales en México. Consejo Civil Mexicano para la Silvicultura Sostenible A.C., Universidad Autónoma Chapingo, Agencia de los Estados Unidos para el Desarrollo Internacional. México. Recuperado de https://www.ccmss.org.mx/acervo/problemas-yoportunidades-en-el-mercado-para-las-empresas-socialesforestales-en-mexico-borrador/

Clark-Tapia, R., Fuente-Carrasco, M. E., Corrado, C.A., Ramos-Morales, M. F., \& Aguirre-Hidalgo, V. (2018). Manejo forestal comunitario y sustentabilidad en Sierra Juárez, Oaxaca. Ed. Fontarama.

Cooperación Alemana [GIZ] (2013). Forestería comunitaria: Un modelo de gestión forestal sostenible $\left(1^{\mathrm{a}} \mathrm{ed}\right.$.). Programa de Fomento al Manejo Sostenible de Recursos Naturales y Desarrollo Económico Local (PRORENA). Recuperado de http://www.chmhonduras.org/phocadownloadpap/CBD/PNB /GIZ/Modelo $\% 20$ de $\% 20$ Gestin $\% 20$ Forestertal $\% 20$ Comunitari a.pdf

Comisión Nacional Forestal [Conafor]. 2008. Programa Institucional 20072012. México. Recuperado de https://snigf.cnf.gob.mx/wpcontent/uploads/2019/02/Programas-Institucional-20072012.pdf

Emery, M., \& Ronald, E.P. (1996). The search conference: a powerful method for planning organizational change and community action. San Francisco: Jossey-Bass Publishers.. Recuperado de file:///C:/Users/C.\%20Luj\%C3\%A1n/Downloads/epdf.pub_t he-search-conference-a-powerful-method-for-planni\%20(1).pdf

Garibay, C. (2007). El dilema corporativo del comunalismo forestal. Desacatos, 23. 251-274.

Giddens, A. (1984). The constitution of society: Outline of the theory of structuration. Cambridge, UK: Policy Press. Recuperado de http://www.communicationcache.com/uploads/1/0/8/8/1088 7248/the_constitution_of_society.pdf

Giménez-Pérez, F. (2008). Antístenes y la democracia. El Catoblepas, 73. Recuperado de http://nodulo.org/ec/2008/n073p12.htm

Goodstein, L. D., Nolan, T. M., \& Pfeiffer, J. W. (2002). Planeación estratégica aplicada. Cómo desarrollar un plan que funcione. México: Mc Graw Hill.

Hodgdon, B., \& Estrada, O. (2015). Hacia una empresa forestal comunitaria integrada. Un estudio de caso del ejido El Largo y Anexos (Chibuabua, Mexico). Fondo Multilateral de Inversiones (FOMIN) y Rainforest Alliance. Estudios de casos de comunidades forestales No. 3/10. Recuperado de https://www.rainforestalliance.org/lang/sites/default/ files/publication/pdf/el-largocase-study-es.pdf 
Instituto Nacional de Estadística, Geografía e Informática [Inegi]. (2016). Resultados de la actualización del marco censal agropecuario 2016. Recuperado https://www.inegi.org.mx/programas/amca/2016/

Janja, E., Gretzinger, S., Camacho, O., Sabogal, C., \& Arce, R. (2016). Desarrollo forestal empresarial por comunidades: guia práctica para promotores forestales comunitarios en los trópicos americanos. Organización de las Naciones Unidas para la Alimentación y la Agricultura (FAO) y el Centro Agronómico Tropical de Investigación y Enseñanza (CATIE). 223 p. Recuperado de http://www.fao.org/3/a-i5984s.pdf

Kandzior, A. (2013). 10 años de acompañamiento y trabajo junto a las comunidades campesinas e indígenas de Chile. Reportaje y Entrevista. Revista Bosque Nativo, 52, 7-21. Recuperado de https://issuu.com/bosquenativo/docs/rbn_52_fullweb

Luján, C., Olivas, J. M., \& Vázquez, S. (2011). Desarrollo forestal integral del Ejido El Largo y Anexos, Mpio. Madera, Chih.: Plan Rector con visión a largo plazo y plan operativo para la puesta en marcha del proyecto. Reporte técnico. Delicias, Chih.

Luján, C., Olivas, J. M., González, H. G., Gómez, O., \& Cuautle, M. A. (2006). Plan Estratégico Participativo para el Desarrollo Forestal Sustentable en el Estado de Chihuahua. Reporte Técnico. Universidad Autónoma de Chihuahua. Cd. Delicias Chih. México

Luján, C., Chapela, G., González, H.G., Olivas, J., \& Vázquez, S. (2018). De la Profortarah a la certificación. Las empresas sociales forestales de Chibuahua, Los ejidos: La Trinidad y Aboreachi: Competitividad de las Empresas Sociales Forestales en México. Universidad Autónoma Chapingo, Consejo Civil Mexicano para la Silvicultura Sostenible A.C., Primera Edición. México.

Luján, C., Olivas, J. M., \& Vázquez, S. (2019). Etapas estructurales del proceso de gestión estratégica para el desarrollo el desarrollo forestal comunitario sustentable. Reporte técnico. Universidad Autónoma de Chihuahua-Facultad de Ciencias Agrícolas y Forestales. Delicias, Chih.

Palomo, I., Martín-López, B., López, C., \& Montes, C. (2010). Hacia un nuevo modelo de gestión del sistema socio-ecológico de Doñana basado en la construcción de una visión compartida sobre sus ecofuturos. Facultad de Ciencias. Edificio de Biología. Madrid, España. Recuperado de https://www.researchgate.net/publication/263090212_El_siste ma_socio-

ecologico_de_Donana_ante_el_cambio_global_Planificacion_de _escenarios_de_eco-futuro\#fullTextFileContent

Pérez-Castañeda, J. C., \& Makinlay, H. (2015). ¿Existe aún la propiedad social agraria en México?. Polis, 11(1), 45-82.

Ramírez, S. (1999). Teoría general de sistemas de Ludwig von Bertalanffy. Universidad Nacional Autónoma de México. Centro de
Investigaciones Interdisciplinarias en Ciencias y Humanidades. Recuperado de http://computo.ceiich.unam.mx/webceiich/docs/libro/Teoria_ General_de_Sistemas_de_Ludwig_Von_Bertalanffy.pdf

Rodríguez-Zúñiga, J., González-Guillén, M. J., \& Valtierra-Pacheco, E. (2019). Las empresas forestales comunitarias en la región de la Mariposa Monarca, México: un enfoque empresarial. Bosque (Valdivia), 40(1), 57-69. doi: 10.4067/S0717-92002019000100057

Sabogal, C., De Jong, W., Pokorny, B., \& Louman, B. (2009). Manejo forestal comunitario en América Latina: experiencias, lecciones aprendidas y retos para el futuro. CIFOR, Indonesia; CATIE-Costa Rica. 274p. Recuperado de http://www.cifor.org/publications/pdf_files/Books/BSabogal0 801S2.pdf

Sánchez-Vidaña, D. L., Valtierra-Pacheco, E., González-Guillén, M. J., \& León-Merino, A. (2018). Capital humano e innovación en el proceso de integración del aprovechamiento forestal maderable en el ejido Gómez Tepeteno, Tlatlauquitepec, Puebla. Madera y Bosques, 24(3), e2431654. doi: 10.21829/myb.2018.2431654.

Secretaría de Gobernación. (2019). Plan nacional de desarrollo 20192024. En el Diario Oficial de la Federación del 12 de julio de 2019. Recuperado de https:/ / www.dof.gob.mx/nota_detalle.php?codigo $=5565599 \& \mathrm{f}$ echa $=12 / 07 / 2019$

Secretaría de Medio Ambiente y Recursos Naturales [Semarnat]Comisión Nacional Forestal [Conafor]. (2019). Programa anual de trabajo 2019. Comisión Nacional Forestal. México. Recuperado de https://www.conafor.gob.mx/transparencia/docs/PAT_2019_ CONAFOR.pdf

Secretaría de Medio Ambiente y Recursos Naturales [Semarnat]. (2014). Programa Nacional Forestal 2014-2018. México: Secretaria de Medio Ambiente y Recursos Naturales. Diario Oficial de la Federación. 28 de abril de 2014. Recuperado de http://www.conafor.gob.mx:8080/documentos/docs/4/5382Pr ograma $\% 20$ Nacional $\% 20$ Forestal $\% 202014-2018 . p d f$

Sepúlveda, S., Rodríguez, A., Echeverría, R., \& Portilla, M. (2003). El enfoque territorial del desarrollo rural. San José, Costa Rica: Instituto Interamericano de Cooperación para la Agricultura. Dirección de Desarrollo Rural Sostenible. Recuperado de https://www.academia.edu/33151738/EL_ENFOQUE_TERR ITORIAL_DEL_DESARROLLO_RURAL_INSTITUTO_IN TERAMERICANO_DE_COOPERACI\%C3\%93N_PARA_L A_AGRICULTURA

Torres-Rojo, J. M., \& Graft-Montero, S. (2014). Desarrollo forestal comunitario: lecciones aprendidas, tendencias y perspectivas. En J. M. Torres (Ed.), Desarrollo forestal comunitario: la política pública $\left(1^{\text {a }}\right.$ 
ed.), Chapter 8. CIDE Editores. Recuperado de http://libreriacide.com/?P=producto\&PRODfamily=libros\&PR ODclassification $=453 \&$ PRODproduct $=91231 \#$. Xou $7 \mathrm{pshKg} 2 \mathrm{w}$

Manuscrito recibido el 11 de septiembre de 2020

Aceptado el 24 de octubre de 2020

Publicado el 21 de abril de 2021
Este documento se debe citar como:

Luján-Álvarez, C., Olivas-García, J. M., Vázquez-Álvarez, S.,

Hernández-Salas, J., \& Castruita-Esparza. L. U. (2021). Sistema de gestión estratégica forestal participativa para el desarrollo forestal sustentable. Madera y Bosques, 27(1), e2712260. doi: 10.21829/myb.2021.2712260

Madera y Bosques por Instituto de Ecología, A.C. se distribuye bajo una Licencia Creative Commons Atribución-NoComercialCompartirlgual 4.0 Internacional. 